\title{
Systematical analysis of nine Chinese patients with progressive pseudorheumatoid dysplasia and assessment of calcitriol treatment
}

Lei Yin ( $\square$ yinleik@hotmail.com )

Shanghai Children's Medical Center https://orcid.org/0000-0003-4795-8710

\section{Youying Mao}

Shanghai Children's Medical Center

Yunfang Zhou

Shanghai Children's Medical Center

yongnian Shen

Shanghai Children's Medical Center

\section{Huijin Chen}

Shanghai Children's Medical Center

Wei Zhou

Shanghai Childdren's Medical Center

Yanliang Jin

Shanghai Children's Medical Center

Hua Huang

Shanghai Children's Medical Center

Yongguo Yu

Shanghai Jiaotong University School of Medicine Xinhua Hospital

Jian Wang

Shanghai Children's Medical Center

Research

Keywords: progressive pseudorheumatoid dysplasia, noninflammatory, articular cartilage, WISP3 gene mutation, calcitriol

Posted Date: June 23rd, 2020

DOI: https://doi.org/10.21203/rs.3.rs-37114/v1

License: (c) (1) This work is licensed under a Creative Commons Attribution 4.0 International License.

Read Full License 



\section{Systematical analysis of nine Chinese patients with progressive pseudorheumatoid}

dysplasia and assessment of calcitriol treatment

Lei Yin ${ }^{\mathrm{a}, \mathrm{b}, \#, *}$, Youying Mao ${ }^{\mathrm{a}, \#}$, Yunfang Zhou ${ }^{\mathrm{b}}$, Yongnian Shen ${ }^{\mathrm{b}}$, Huijin Chen ${ }^{\mathrm{b}}$, Wei Zhou ${ }^{\mathrm{a}}$, Yanliang Jin ${ }^{\mathrm{c}}$, Hua Huang ${ }^{\mathrm{c}}$, Yongguo Yu ${ }^{\mathrm{d}}$, Jian Wang, ${ }^{\mathrm{e}}$,

${ }^{a}$ Department of Nephrology, Shanghai Children's Medical Center, Shanghai Jiao tong University School of Medicine, Shanghai, 200127, China

b Special Consultation Clinic for rare and complicated diseases, Shanghai Children's Medical Center, Shanghai Jiao tong University School of Medicine, Shanghai, 200127, China

C Department of Rheumatology, Shanghai Children's Medical Center, Shanghai Jiao tong University School of Medicine, Shanghai, 200127, China d Department of Pediatric Endocrinology, Xinhua hospital, Shanghai Jiao tong University School of Medicine, Shanghai, 200127, China

e Department of Medical Genetics and Molecular Diagnostic Laboratory, Shanghai Children's Medical Center,

Shanghai Jiao tong University School of Medicine, Shanghai, 200127, China

\# The first two authors have contributed equally to this study.

* Correspondence to: Dr. Lei Yin (yinleik@hotmail.com), Department of Nephrology, Shanghai Children's Medical Center, Shanghai Jiaotong University School of Medicine. 1678 Dongfang Road, Shanghai 200127, P.R China; Fax 86-21-58756923 or

Dr. Jian Wang (Labwangjian@shsmu.edu.cn), Department of Medical Genetics and Molecular Diagnostic Laboratory, Shanghai Children's Medical Center, Shanghai Jiaotong University School of Medicine. 1678 Dongfang Road, Shanghai 200127, P.R China; Fax 86-21-58756923.

\section{Conflict of interest}

The authors declare that they have no conflict of interests. 


\section{ABSTRACT}

Background: Progressive pseudorheumatoid dysplasia (PPRD) (MIM 208230) is a rare genetic disease characterized by progressive noninflammatory arthropathy affecting primarily the articular cartilage. Most patients are initially misdiagnosed because of the rarity of the disease and lack of awareness by most clinicians. Therefore, the purpose of the present study was to provide further diagnostic options to help clinicians make early precise diagnosis, and to investigate a new potential treatment for patients with PPRD.

Methods: Clinical manifestations, radiographic features, Sanger Sequencing to determine WISP3 gene mutation and follow-up records were collected in 9 Chinese patients with PPRD. The time until diagnosis, relationship between clinical phenotypes and genotypes, and treatment effects were analyzed.

Results: The clinical history and characteristics, mutations in the WISP3 gene, radiological data, treatment and outcome of 9 PPRD children were collected and reviewed. There were 3 pairs of siblings in this group and one patient had parental consanguinity. Five patients were misdiagnosed with juvenile idiopathic arthritis (JIA). The onset of disease in most patients (8/9) was between 3 and 6 years of age. The interval between onset of symptoms and obtaining the diagnosis for 8 of the patients varied between 3.6 years to 20 years. The onset of symptoms included enlarged and stiff interphalangeal joints of the fingers, gait disturbance or joint pain. Blood analysis revealed normal range of inflammatory parameters (erythrocyte sedimentation rate, Creactive protein). Serum levels of 25-hydroxyvitamin D3 were analyzed in six patients and ranged from 6.16 to $22.1 \mathrm{ng} / \mathrm{ml}$, all lower than the normal range. Radiographic findings included different degree of abnormal vertebral bodies, epiphyseal enlargement of the interphalangeal joints with juxta-articular osteopenia, or reduction in hip articular space and cyst-like structures femoral head. A total of 7 variants in the WISP 3 gene were identified in the 9 cases, presenting with three homozygous variants (c.397_404delCAAGTGTT, c.667T $>$ G, and c.589+2T>C) and three compound heterozygous variants $\quad(\mathrm{c} .589+2 \mathrm{~T}>\mathrm{C} / \mathrm{c} .667 \mathrm{~T}>\mathrm{G}, \quad$ c.624dupA $/ \mathrm{c} .756 \mathrm{C}>\mathrm{A}, \quad$ and c. $646 \mathrm{~T}>\mathrm{C} / \mathrm{c} .1000 \mathrm{~T}>\mathrm{C}$ ). After the treatment of calcitriol in the 6 patients with low level 
of 25-hydroxyvitamin D3 for 1.25 years to 1.75 years, two cases showed stable clinical disease activity and the other four patients joints' pain and abnormal gait had improved. Conclusions: Combining the patient's family history, clinical features presenting with abnormal gait or enlarged and stiff interphalangeal joints of the fingers, normal inflammatory markers, and the characteristic radiographic findings, we can obtain the clinical diagnosis of PPRD for the patients at a very early stage of the disease. The patients with PPRD having c.624dupA mutation in WISP3 may have delayed onset. Calcitriol showed treatment benefit by improving symptoms and decelerating progression of PPRD disease.

Keywords: progressive pseudorheumatoid dysplasia; noninflammatory; articular cartilage; WISP3 gene mutation; calcitriol 


\section{Introduction}

Progressive pseudorheumatoid dysplasia (PPRD) (MIM 208230) is a rare genetic disease initially described by Wynne-Davies et al in $1982^{1}$. This autosomal recessive inherited skeletal dysplasia is characterized by progressive noninflammatory arthropathy affecting primarily the articular cartilage ${ }^{2}$. The prevalence of PPRD has been estimated at one per million in the United Kingdom ${ }^{3}$, but it may be underdiagnosed due to the overlap of clinical and imaging features with other pediatric musculoskeletal disorders, such as juvenile idiopathic arthritis (JIA), Scheuermann's disease, spondyloepiphyseal dysplasia, Blount disease, Stickler syndrome, and mucopolysaccharidosis ${ }^{4,5}$. The onset of symptoms is usually in childhood between 3 to 6 years of age, and symptoms progressively worsen with time ${ }^{2}$. As the disease is so rare and clinicians are thus lacking awareness, most patients are initially misdiagnosed ${ }^{6}$. The average delay in diagnosis is 6 to 13 years $^{2}$. The patients are most often referred to a pediatrician, rheumatologist, orthopedic surgeon, and very few to a medical geneticist ${ }^{7}$. PPRD is caused by mutations in the WISP3 gene which maps to chromosome 6q22, and encodes a protein in the connective tissue growth factor that is expressed in synoviocytes and chondrocytes involved in bone and cartilage growth and development ${ }^{8}$. Homozygous or compound heterozygous WISP3 mutations cause the loss of articular cartilage, which leads to the progressive narrowing of all articular spaces and reduced joint mobility ${ }^{9}$. While this molecular basis was elucidated over 10 years ago, no progress has been made towards a specific treatment for PPRD ${ }^{2}$. $1,25(\mathrm{OH})_{2} \mathrm{D}_{3}$ (calcitriol), which is used to treat hypocalcemia, osteoporosis, and prevention of corticosteroid-induced osteoporosis ${ }^{10}$, has showed chondro-protective effects in human chondrocytes ${ }^{11}$. Combined with the results of a murine model to study the role of $1,25(\mathrm{OH})_{2} \mathrm{D}_{3}$, the patients and rat model with rheumatoid arthritis, and the properties of patient's cartilage and chondrocytes under PPRD, we suspected that calcitriol may maintain the normal proliferation of articular chondrocytes by accelerating chondrocyte apoptosis ${ }^{12-14}$ and could thus be used to treat PPRD. In the present study, we systematically evaluated the clinical history and characteristics, radiological data and WISP3 gene mutations in 9 patients with PPRD recruited by our 
special consultation clinic, and evaluated potential treatment with calcitriol for PPRD. Furthermore, based on the information between the age of onset and genotype of the patients, we suggest that patients with PPRD with underlying c.624dupA mutation in the WISP3 gene may have delayed onset.

\section{Materials and Methods}

\section{Patients}

Research subjects' blood samples and pedigree data were obtained from August 2011 to December 2019 from individuals with a genetic confirmed diagnosis of progressive pseudorheumatoid dysplasia in our center. According to the principles of the declaration of Helsinki, informed written consent was obtained from the patients and their parents. This study was approved by the Ethics Committee of the Shanghai Children's Medical Center, Shanghai Jiao tong University School of Medicine, Shanghai, China. The medical history, results of physical examination, other relevant laboratory investigations and radiological data were obtained from the patients during clinical visits/routine clinical care and systematically analyzed. Venous blood samples were collected from both the patients and their parents.

\section{Whole blood analysis}

Blood tests of inflammatory parameters were performed including complete blood count, and C-reactive protein (CRP), erythrocyte sedimentation rate (ESR) and the tests to exclude other diseases including liver function test, serum blood urea and creatinine, rheumatoid factor, antinuclear antibody, HLA-B27, electrolytes, thyroid function tests, parathyroid hormone.

\section{Sanger Sequencing}

Genomic DNA was extracted from 2-mL peripheral blood samples using the QIAamp Blood DNA Mini Kit® (Qiagen, Hilden, Germany) according to the manufacturer's instructions. All 5 coding exons and the exon-intron boundaries of the WISP3 gene 
(NM_003880.3) were amplified by polymerase chain reaction (PCR) using the primers described preciously ${ }^{15}$. Sanger sequencing was performed on an Applied Biosystems ABI3730XL sequencer (Thermo Fisher Scientific, Waltham, MA, USA), and the data were then analyzed using Mutation Surveyor v.4.0.4 software (SoftGenetics LLC, State College, PA, USA).

\section{Results (table 1$)$}

Nine patients (two males and 7 females, 7 children and 2 adults) were referred to our center for diagnostic assistance by local hospitals from 4 provinces of China during the period of August 2011 to December 2019. Five of them (case3, 4, 6, 7, 9) were initially diagnosed with juvenile idiopathic arthritis (JIA). Case9 had been treated with methotrexate and cyclophosphamide for 3 months without improvement. Case 5 was diagnosed with ankylosing spondylitis because of positive HLA-B27. Case 8 had the suspected diagnosis of congenital spondyloepiphyseal dysplasia. Six patients (case1 and 2, case 3 and 4, case6 and 7) were 3 pairs of siblings. Case5 had parental consanguinity. After review of the family histories, clinical symptoms, and laboratory investigations of the patients, there were several clues which guided us to the diagnosis of PPRD. Key indicators were the lack of increased inflammatory parameters in the blood (erythrocyte sedimentation rate, C-reactive protein) (table1) and the radiological findings of the abnormal vertebral bodies (Figure 1c, 2c). The patients and their parents were subjected to DNA sequencing of WISP3 to investigate the genetic cause of this anomaly and for appropriate genetic counseling.

\section{History of diagnosis and clinical findings}

Eight of the nine patients had onset of symptoms in childhood between 3 and 6 years of age. Only case 8 began to have the symptoms from 14 years old. The interval between onset of symptoms and obtaining the diagnosis with PPRD for all patients except case2 varied between 3.6 years to 20 years. Case 2 got the definite diagnosis in one month without any joint enlargement or movement limitation (Figure1a, 1b) since the patient's older brother (case1) had already been diagnosed with PPRD. The onset of symptoms 
for the observed patients included enlarged and stiff interphalangeal joints of the fingers (2/9), gait disturbance (5/9) or joint pain (4/9). Causes for clinic-visits were the worsening of symptoms, most (7/9) had progressive abnormal gait, one patient had short stature, and one patient visited the clinic for further investigation upon her brother's positive PPRD diagnosis. At time of diagnosis, all of the patients except case2 had manifested enlargement of the interphalangeal joints of the fingers (Figure2a, 3a) and varying degree of limitation of joint movement, especially for the fingers and hips (Figure $2 \mathrm{e}, 2 \mathrm{f}, 3 \mathrm{~b}$ ). No signs or symptoms of inflammatory arthritis such as redness, heat, effusion, or soft tissue swelling were observed. Six patients had gait disturbance and two patients had progressed to walking difficulties that required the aid of crutches (Figure 3b) or wheels.

Whole blood analysis

All the patients' ESR and CRP levels were in normal range. The normal range of ESR and CRP were lower than $20 \mathrm{~mm} / \mathrm{hr}$ and $8 \mathrm{mg} / \mathrm{L}$, respectively. The normal range of serum level of 25-hydroxyvitamin D3 was higher than 30ng/ml(table1). Their liver function test, serum blood urea and creatinine, rheumatoid factor, antinuclear antibody, HLAB27, electrolytes, thyroid function tests, parathyroid hormone were all normal (data not shown) except for case5 who had positive HLA-B27. After we found that case2 had low serum level of 25-hydroxyvitamin D3, we also analyzed this parameter in case1, case3, case6, case8 and case9. The results of serum level of 25-hydroxyvitamin D3 ranged from 6.16 to $22.1 \mathrm{ng} / \mathrm{ml}$, all below normal.

\section{Radiographic features}

All the patients' radiographs were collected and analyzed except case7, where radiographs could not be obtained. All (8/8) patients had different degree of abnormal vertebral bodies, the severity ranging from platyspondyly and anterior blunt of the vertebral bodies (Figure 1c) to anterior beak-like of the vertebral bodies (Figure 2c). From four patients, hand X-rays were obtained which consistently showed epiphyseal enlargement of the interphalangeal joints with juxta-articular osteopenia (Figure 2b). 
Other radiological findings including osteopenia, reduction in hip articular space (Figure 2d), and cyst-like structures in the left femoral head (Figure 3c).

\section{WISP3 variants}

We identified 7 different WISP3 variants ( 3 missense, 3 truncating, and 1 splicing) in the patients (Table 2). Case1 and 2 had a novel homozygous variant of c.397_404del CAAGTGTT (p.Gln133Serfs*34). Case 3 and 4 had a compound heterozygous variant for c. $589+2 \mathrm{~T}>\mathrm{C}^{16}$ and c.667T $>\mathrm{G}$ (p.Cys223Gly) ${ }^{17}$. Case 5 had the homozygous variant of c.667T $>$ G (p.Cys223Gly) ${ }^{17}$. Case 6 and 7 had a compound heterozygous variant for c.646T $>$ C (p.Trp216Arg) and c.1000T $>$ C (p.Ser334Pro) $)^{16,18}$. Case 8 had compound heterozygous variant of c.624dupA, (p.Cys209Metfs*21) ${ }^{15,19,20}$ and c.756C $>$ A (p.Cys $252 *)^{17}$. Case 9 had homozygous variant of c. $589+2 \mathrm{~T}>\mathrm{C}^{16}$.

Treatment and outcomes

After obtained the definite diagnosis of PPRD, the patients were treated with physical therapy and occasional NSAIDs for pain relieve. Upon finding low serum level of 25hydroxyvitamin D3 in six patients, combined with their normal serum calcium, phosphate, alkaline phosphatase, parathyroid hormone and osteopenia, they began treatment with oral calcitriol for 1.25 years to 1.75 years. After the treatment of calcitriol, case2 proceeded without showing any symptoms, while case6 neither improved nor worsened. The other 4 patients' joint pain, the stiffness and restricted movements of their joints were improving gradually during the treatment instead of getting worse as before. In addition, case 8 was treated with left hip arthroplasty at 32year age and right hip arthroplasty at 35-year age. Case7 was planning to receive hip arthroplasty.

\section{Discussion}

PPRD is a rare and inheritable skeletal dysplasia ${ }^{21}$. The clinical and radiological phenotype of patients with PPRD is rather homogeneous with progressive worsening 
of symptoms and radiological changes during childhood and adolescence ${ }^{2}$. The unspecific symptoms often cause a significant delay in diagnosis ${ }^{2}$, or even lead to misdiagnosis resulting in incorrect treatment ${ }^{22}$. In our nine patients with PPRD presented here, five were misdiagnosed with juvenile idiopathic arthritis (JIA) before they came to our center, and one patient (case9) even had received treatment with immunosuppressants despite their autoimmunity markers and inflammatory parameters all within normal range. Moreover, in the two pair of sisters (case3 and 4, case6 and 7), when the younger sisters (case3, case6) were diagnosed with PPRD, their older sisters had already suffered from symptoms for 3 or 11 years, respectively. One patient was misdiagnosed with ankylosing spondylitis only because of positive HLA-B27. Her parental consanguinity, normal inflammatory parameters and cyst-like structures in the left femoral head were all neglected. Most of our patients (8/9) had the onset of symptoms between 3 and 6 years of age, which is consistent with a previous review including large samples concerning age at onset ${ }^{2}$. Diagnosis of PPRD is often delayed, sometimes for decades because few clinicians are aware of the disease ${ }^{9}$. The interval between the onset and obtaining the definite diagnosis for most of our patients (7/9) was longer than 6 years. Most of our patients presented with the characteristic manifestations of PPRD including gait abnormalities, fatigability, symmetric stiffness in multiple joints (particularly the hip), and enlarged interphalangeal joints of the hands 9 . Radiological features of PPRD include spondyloepiphyseal dysplasia varied from platyspondyly with loss or narrowing of intervertebral disc spaces, and anterior blunt of the vertebral bodies to anterior beak-like of the vertebral bodies, enlarged and flattened capital femoral epiphyses and short and wide femoral necks, enlarged epiphyses, loss of or narrow joint spaces in the metacarpophalangeal and interphalangeal joints and osteopenia ${ }^{2,3}$. The early radiological signs are metaphyseal enlargement of interphalangeal joints ${ }^{2}$. In our patients, we found that anterior blunt of the vertebral bodies also could be an early radiological sign when the patient (case2) has not developed obvious clinical symptoms and characteristics yet. If the family history, clinical features presenting with abnormal gait or enlarged and stiff interphalangeal joints of the fingers but normal inflammatory markers could be 
combined with the characteristic radiographic findings, all the patients could get the clinical diagnosis of PPRD at very early stage of the disease.

PPRD is due to a mutation in Wnt1-inducible signaling protein 3 (WISP3) gene, which encodes a signaling factor involved in cartilage homeostasis and bone growth by promoting type II collagen and aggrecan expression and regulating chondrocyte proliferation and differentiation ${ }^{9}$. WISP3 expression is decreased in PPRD articular chondrocytes (ACs). PPRD ACs are characterized by increased proliferation activity and decreased apoptosis and may account for the metaphyseal enlargement seen in PPRD radiographs ${ }^{23}$. They are highly mineralized and significantly stiffer than those from normal articular cartilage which may slow down cartilage growth and repair $^{14}$. All of our nine patients had WISP3 mutations. Four patients (case1, 2, 5, 9), including a pair of brother and sister (case1 and 2), and case5 having parental consanguinity, had homozygous mutations of the WISP3 gene. Five patients (case3, 4, 6, 7,8) including two pairs of sisters (case 3 and 4, case6 and 7) had compound heterozygous mutations of the WISP3 gene. While there is a low prevalence for PPRD in the UK, previous reports indicate it is more common in the Middle East, Gulf States, Arabic countries, and countries of the Mediterranean basin, possibly because of the high consanguinity rate in these populations ${ }^{2,4,8}$. Case 5 had the homozygous missense mutation c.667T $>\mathrm{G}$, p.C223G in the WISP3 gene. Three of our patients had WISP3 mutations involving c. $589+2 \mathrm{~T}>\mathrm{C}$, showing a homozygous mutation or compound heterozygous mutation with c. $667 \mathrm{~T}>\mathrm{G}$. The c.667T $>\mathrm{G}$ mutation, located in exon 4 of the WISP3 gene has been reported before in Chinese and other ethnic origins' children affected with PPRD ${ }^{8,22}$. Exon4 encodes a thrombospondin type 1 domain (TSP-1) with six cysteine residues, which plays a vital role in Notch signaling that regulates chondrocyte differentiation and is also implicated in ossification and osteoarthritis, suggesting its importance in the functionality of $W I S P 3^{22}$. The c. $589+2 \mathrm{~T}>\mathrm{C}$ mutation has been reported only in Chinese children with PPRD and is located in intron 4 of the WISP3 gene $^{16}$. The mutation c.624dupA has been reported in patients from China, India and countries of North and West Africa ${ }^{15,19,24}$. This duplication mutation is also located in exon 4 of WISP3. The frameshift mutation leads to the substitution of the cytosine with Methionine at position 
209, creating a new reading frame which introduces a premature stop codon 21 residues downstream $^{24}$. The ages of onset of three African patients with PPRD having homozygous mutation (c.624dupA) in WISP3 were much older (from 10-year old to 18-year old) than the common onset age of PPRD ${ }^{24}$. The ages of onset of two Chinese patients with compound heterozygous mutations (c.624dupA, c.756C $>$ A) were also older than common range, 7 - and 8-year old ${ }^{15}$. In line with this, case8, who presented with a compound heterozygous mutation c.624dupA, c.756C $>$ A, had late and atypical symptoms with onset at 14-years. Furthermore, the compound heterozygous mutation (c.756C $>$ A, c.667T $>$ G) was previously reported in one pair of Chinese siblings. The ages of onset of this sibling were 6-year old and 3-year old ${ }^{17}$. The onset age of our case 5 having a homozygous missense mutation (c.667T $>$ G, p.C223G) was 6-year old. Based on the information of the age of onset and genotype of the patients, we suspect that the patients with PPRD having c.624dupA mutation in WISP3 may have delayed onset. One of our pair of sisters (case6 and 7) had a compound heterozygous mutation (c.646T $>$ C, c.1000T $>$ C). The mutation in c.646T $>$ C (p.Trp216Arg) has not been reported previously and its allele frequency was $0.00081 \%$ in gnomAD database. The missense mutation (c.1000T.C/p. S334P) has, however, been reported in Chinese patients $^{16,18}$. It's located in exon 6 of WISP 3 and induces a transition of a single nucleotide that caused the amino acid serine (TCT) in codon 334 to change to proline $(\mathrm{CCT})^{16}$. Ser334Pro mutant WISP3 protein was detected the C-terminal peptide towards the opposite direction, 840delT mutation of WISP3 gene coexistence of T1000C substitution is necessary for the clinical onset of PPRD. The patient with this compound heterozygous mutation presented with marked abnormalities of cartilage chondrocytes and matrix in morphology and function. ${ }^{25}$. The other pair of brother and sister in our patients (case1 and 2) had the homozygous mutation (c.397_404del CAAGTGTT) in WISP3 gene. It has not been reported previously and could be classified as pathogenic variation analyzed by Polymorphism Phenotyping v2, sorting intolerant from tolerant (SIFT) databases and combined annotation-dependent depletion (CADD) scores that predict the gene mutation effect. Further study is needed. Although the molecular basis of PPRD has been elucidated for more than 10 years, no 
progress has been made towards a specific treatment ${ }^{2}$. The current treatment options only alleviate symptoms. Pain due to secondary osteoarthritis may respond to NSAIDs. Physical therapy and rehabilitation may help preserve joint mobility. Surgical intervention, including realignment of the lower limbs, joint arthroplasty, and/or treatment of spinal stenosis when necessary, are the mainstay of treatment today ${ }^{3,5}$. Total hip arthroplasty is a treatment option for PPRD patients to restore the joint function and improve life quality. It has been shown that THA is an effective treatment for PPRD patients with hip arthropathy, with satisfactory clinical and radiological outcome after follow-up for 18 months to 93 months (mean 47.9 months) ${ }^{20}$. Case 8 has received bilateral hip arthroplasty and since has been able to continue his job as a driver. The other adult patient is preparing to receive this treatment.

$1,25(\mathrm{OH})_{2} \mathrm{D}_{3}$ (calcitriol), works on the key role of in calcium homeostasis and bone mineralization, which is actually the most active form of vitamin $\mathrm{D}^{26}$. Calcitriol has been prescribed for the treatment of hypocalcemia, osteoporosis, and prevention of corticosteroid-induced osteoporosis so far ${ }^{10}$. It has a very short half-life and circulates in the serum at concentrations that are about $0.1 \%$ of those of the prohormone $25(\mathrm{OH}) \mathrm{D}_{3}$ and its synthesis is tightly regulated by the endocrine system. For these reasons $1,25(\mathrm{OH})_{2} \mathrm{D}_{3}$ levels in the serum are not used to evaluate the vitamin $\mathrm{D}$ status in humans, and $25(\mathrm{OH}) \mathrm{D}_{3}$ is favored as the best indicator of vitamin $\mathrm{D}$ status ${ }^{26}$. Recently, it has been demonstrated that $1,25(\mathrm{OH})_{2} \mathrm{D}_{3}$ accelerates chondrocyte apoptosis by impairing activation of the NF- $\kappa B$ signaling pathway in rats with rheumatoid arthritis ${ }^{12}$. The murine model also showed that $1,25(\mathrm{OH})_{2} \mathrm{D}_{3}$ controlled chondrocyte proliferation and differentiation directly ${ }^{13}$. Concerning their normal liver function, serum creatinine, serum calcium, phosphate, alkaline phosphatase, parathyroid hormone, we treated our patients that presented with low serum level of 25-hydroxyvitamin D3 with oral calcitriol and in the follow-up appointment found that $2 / 6$ patient's symptoms stopped progressing, while 4/6 patient's symptoms improved gradually. We suspected that calcitriol may maintain the normal proliferation of articular chondrocytes by accelerating chondrocyte apoptosis ${ }^{11-13}$ in patients with PPRD. Therefore, our preliminary data with six patients suggest that calcitriol may provide a new beneficial 
treatment option for PPRD. However, more cases will have to be included into the study and long-term follow up is needed for a more comprehensive analysis of the effectiveness of calcitriol in PPRD.

\section{Conclusion}

PPRD is a rare disease characterized by progressive noninflammatory arthropathy affecting primarily the articular cartilage. Despite symptoms severely affecting quality of life, no current treatment consensus exists. In this study we found that calcitriol, which is used in osteoporosis, significantly improved clinical symptoms in 4/6 patients with PPRD. These findings suggest a great potential for implementing calcitriol in the treatment of this disease, and further study with larger case numbers are needed to validate these findings. Furthermore, we observed a connection between the c.624dupA mutation in WISP3 and delayed onset of PPRD symptoms. These findings may aid in earlier diagnosis in such patients.

\section{References}

1. Wynne-Davies R, Hall C, Ansell BM. Spondylo-epiphysial dysplasia tarda with progressive arthropathy. A "new" disorder of autosomal recessive inheritance. The Journal of bone and joint surgery. British volume. 1982;64(4):442-445.

2. Garcia Segarra N, Mittaz L, Campos-Xavier AB, et al. The diagnostic challenge of progressive pseudorheumatoid dysplasia (PPRD): a review of clinical features, radiographic features, and WISP3 mutations in 63 affected individuals. American journal of medical genetics. Part C, Seminars in medical genetics. Aug 15 2012;160C(3):217-229.

3. Bhavani GSL, Shah H, Shukla A, Dalal A, Girisha KM. Progressive Pseudorheumatoid Dysplasia. In: Adam MP, Ardinger HH, Pagon RA, et al., eds. GeneReviews((R)). Seattle (WA)2015.

4. Pomeranz CB, Reid JR. Progressive pseudorheumatoid dysplasia: a report of three cases and a review of radiographic and magnetic resonance imaging 
findings. Skeletal radiology. Sep 2019;48(9):1323-1328.

5. Giray E, Yagci I, Elcioglu HN. Progressive pseudorheumotoid dysplasia: A presentation of four cases with slow and rapid progression and effects of early rehabilitation program. Turkish journal of physical medicine and rehabilitation. Sep 2019;65(3):290-297.

6. Neerinckx B, Thues C, Wouters C, Lechner S, Westhovens R, Van Esch H. A homozygous deletion of exon 1 in WISP3 causes progressive pseudorheumatoid dysplasia in two siblings. Human genome variation. 2015;2:15049.

7. Pode-Shakked B, Vivante A, Barel O, et al. Progressive Pseudorheumatoid Dysplasia resolved by whole exome sequencing: a novel mutation in WISP3 and review of the literature. BMC medical genetics. Mar 29 2019;20(1):53.

8. Sailani MR, Chappell J, Jingga I, et al. WISP3 mutation associated with pseudorheumatoid dysplasia. Cold Spring Harbor molecular case studies. Feb $2018 ; 4(1)$.

9. Torreggiani S, Torcoletti M, Campos-Xavier B, et al. Progressive pseudorheumatoid dysplasia: a rare childhood disease. Rheumatology international. Mar 2019;39(3):441-452.

10. Lung BE, Komatsu DEE. Calcitriol. StatPearls. Treasure Island (FL)2020.

11. Avcioglu G, Ozbek Iptec B, Akcan G, et al. Effects of 1,25-Dihydroxy vitamin D3 on TNF-alpha induced inflammation in human chondrocytes and SW1353 cells: a possible role for toll-like receptors. Molecular and cellular biochemistry. Jan 2020;464(1-2):131-142.

12. Tian $\mathrm{R}$, Li $\mathrm{X}$, Li Y, Wang $\mathrm{K}$, Wang $\mathrm{C}$, Yang $\mathrm{P}$. 1,25(OH)2D3 promotes chondrocyte apoptosis and restores physical function in rheumatoid arthritis through the NF-kappaB signal pathway. Biomedicine \& pharmacotherapy $=$ Biomedecine \& pharmacotherapie. Oct 2018;106:149-155.

13. Hirota $\mathrm{Y}$, Nakagawa K, Mimatsu S, et al. Nongenomic effects of 1alpha,25dihydroxyvitamin D3 on cartilage formation deduced from comparisons between Cyp27b1 and Vdr knockout mice. Biochemical and biophysical research communications. Jan 29 2017;483(1):359-365. 
14. Lin LJ, Ge YM, Tian Y, et al. Multi-scale mechanical investigation of articular cartilage suffered progressive pseudorheumatoid dysplasia. Clinical biomechanics. Jan 22020.

15. Ye J, Zhang HW, Qiu WJ, et al. Patients with progressive pseudorheumatoid dysplasia: from clinical diagnosis to molecular studies. Molecular medicine reports. Jan 2012;5(1):190-195.

16. Sun J, Xia W, He S, et al. Novel and recurrent mutations of WISP3 in two Chinese families with progressive pseudorheumatoid dysplasia. PloS one. 2012;7(6): e38643.

17. Luo H, Shi C, Mao C, et al. A novel compound WISP3 mutation in a Chinese family with progressive pseudorheumatoid dysplasia. Gene. Jun 10 2015;564(1):35-38.

18. Sertedaki A, Papadimitriou A, Voutetakis A, Dracopoulou A, Maniati-Christidi M, Dacou-Voutetakis C. Gene symbol: TSH B. Disease: congenital hypothyroidism. Human genetics. Jul 2004;115(2):174.

19. Bhavani GS, Shah H, Dalal AB, et al. Novel and recurrent mutations in WISP3 and an atypical phenotype. American journal of medical genetics. Part A. Oct 2015;167A(10):2481-2484.

20. Feng B, Xiao K, Ren Y, et al. Mid-Term Outcome of Total Hip Arthroplasty in Patients With Progressive Pseudorheumatoid Dysplasia. Journal of clinical rheumatology : practical reports on rheumatic \& musculoskeletal diseases. Dec 242019.

21. Hu Q, Liu J, Wang Y, et al. Delayed-onset of progressive pseudorheumatoid dysplasia in a Chinese adult with a novel compound WISP3 mutation: a case report. BMC medical genetics. Dec 15 2017;18(1):149.

22. Al Kaissi A, Kenis V, Jemaa LB, et al. Skeletal phenotype/genotype in progressive pseudorheumatoid chondrodysplasia. Clinical rheumatology. Feb 2020;39(2):553-560.

23. Zhou $\mathrm{HD}, \mathrm{Bu} \mathrm{YH}$, Peng YQ, et al. Cellular and molecular responses in progressive pseudorheumatoid dysplasia articular cartilage associated with 
compound heterozygous WISP3 gene mutation. Journal of molecular medicine. Sep 2007;85(9):985-996.

24. Chouery E CS, Dahmen J, Zouari L, Gribaa M, Leban N, Chibani B. Progressive pseudorheumatoid dysplasia in North and West Africa: clinical description in ten patients with mutations of WISP3. Egypt $J$ Med Hum Genet. 2017;18(3):299-303.

25. Peng YQ, Liao EY, Gu HM, et al. [Pathology and molecular pathogenesis of spondyloepiphyseal dysplasia tarda with progressive arthropathy caused by compound CCN6 heterogeneous gene mutations]. Zhonghua yi xue za zhi. Nov 2 2004;84(21):1796-1803.

26. Brandi ML. Indications on the use of vitamin D and vitamin D metabolites in clinical phenotypes. Clinical cases in mineral and bone metabolism : the official journal of the Italian Society of Osteoporosis, Mineral Metabolism, and Skeletal Diseases. Sep 2010;7(3):243-250. 


\section{Declarations}

\section{Ethics approval and consent to participate}

This study has been performed in accordance with the Declaration of Helsinki and the current ethical polices and has been approved by the Ethics Committee of the Shanghai Children's Medical Center (SCMCIRB-K2016013), Shanghai Jiao tong University School of Medicine, Shanghai, China.

\section{Consent for publication}

Not applicable

\section{Availability of data and materials}

All the material and data of the study are available upon request.

\section{Competing interests}

The authors declare that they have no competing interests.

\section{Funding}

Not applicable

\section{Authors' contributions}

LY: designed the study, drafted the initial manuscript, wrote final manuscript, data collection, coordinated and supervised laboratory investigations, data analysis, and patient assessment. YYM: performed the literature search, conceptualized the study, collected, analyzed and interpreted the data, drafted and revised the manuscript. YFZ: communicated with the patients, collected and interpreted clinical data, and worked on manuscript. YNS: communicated with the patients, collected and interpreted clinical data, interpretation and critically reviewed the manuscript. HJC: performed the literature search, conceptualized the study, interpretation and critically reviewed the manuscript. WZ: communicated with the patients, collected and interpreted clinical 
data, and worked on manuscript. YLJ: communicated with the patients, collected and interpreted clinical data, and worked on manuscript. HH: communicated with the patients, collected and interpreted clinical data, and worked on manuscript. YGY: communicated with the patients, collected and interpreted clinical data, supervised laboratory investigations, contributed to data analysis. JW: coordinated and supervised laboratory investigations, contributed to data analysis, critically reviewed the manuscript, wrote final manuscript. All authors read and approved the final manuscript.

\section{Acknowledgements}

We thank Dr. Emely Verweyen (Cincinnati Children's Hospital Medical Center, Ohio, U.S.) for revising the paper sentence by sentence. 
Figures
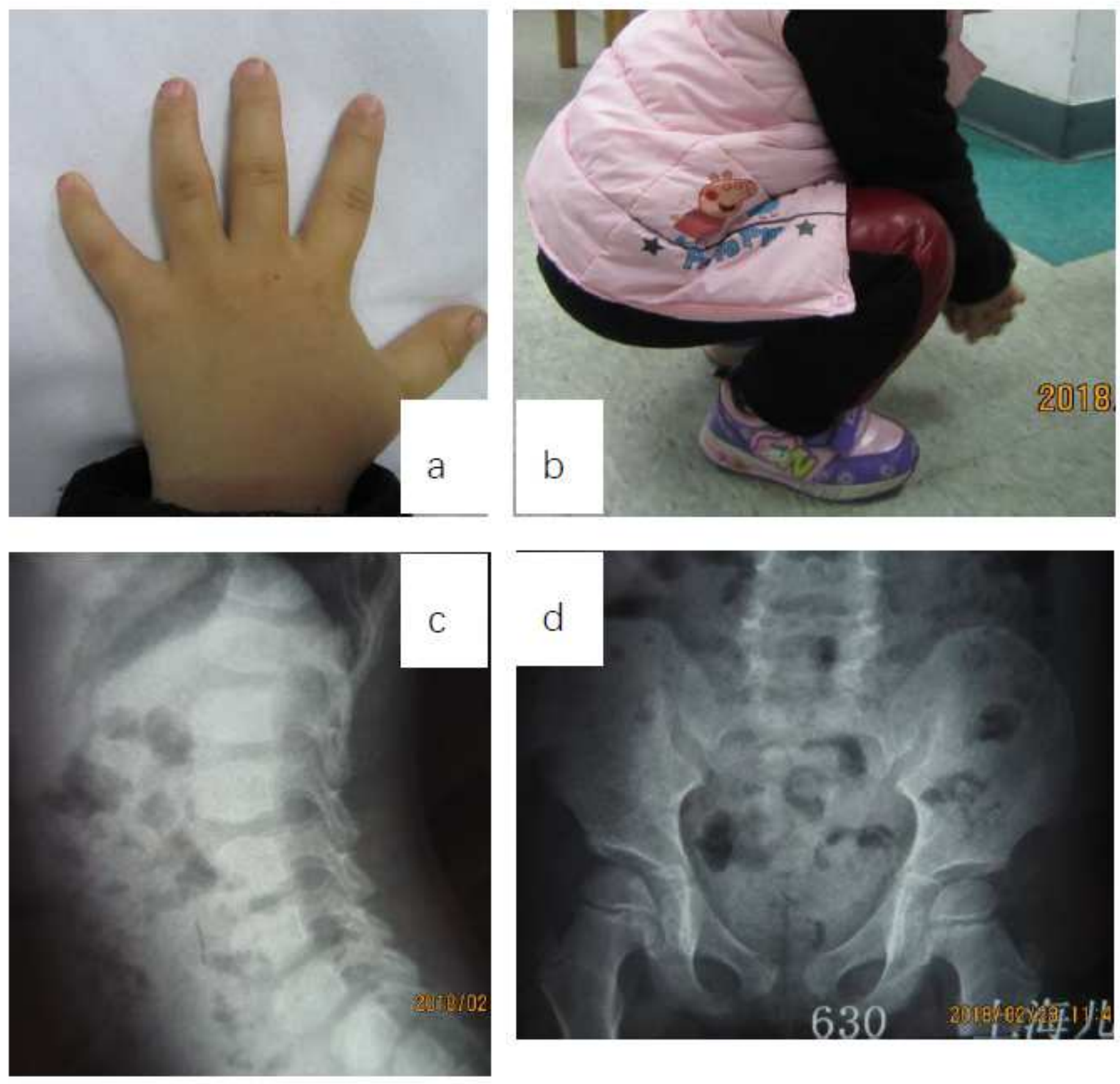

Figure 1

Images of case 2 (girl, 4 years) a. Normal fingers b. Normal squatting c. Radiograph of the thoracolumbar spine showing platyspondyly and anterior blunt of the vertebral bodies $\mathrm{d}$. normal radiograph of pelvis 

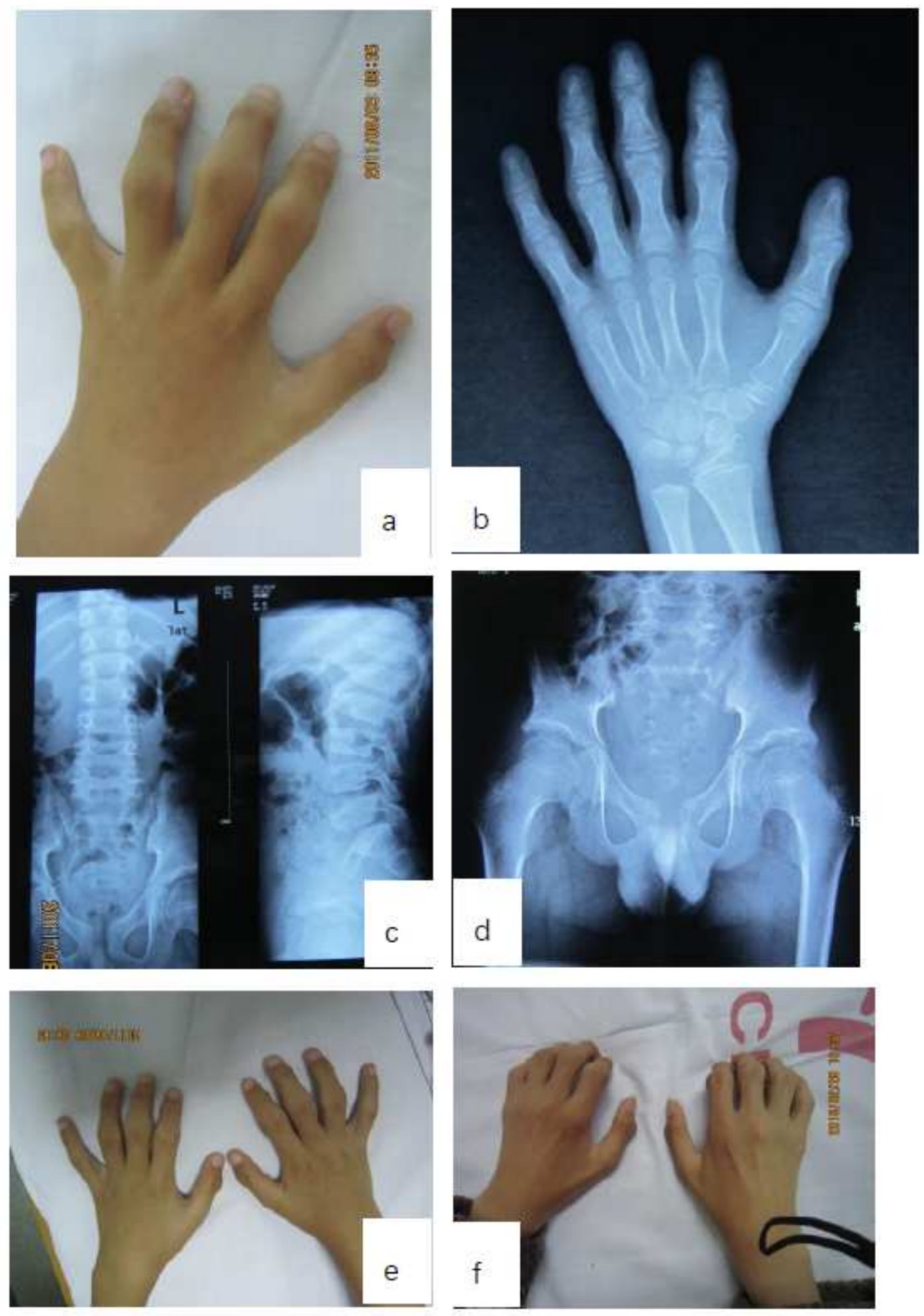

\section{Figure 2}

Images of case 1 (boy, 9 a. E nlarged interphalangeal joints of the fingers $b$. R adiograph of left hand showing epiphyseal enlargement of the interphalangeal joints with juxta articular osteopenia $\mathrm{c}$. Radiograph of the thoracolumbar spine show ing platyspondyly and anterior beak like of the vertebral bodies $d$ Radiograph of pelvis show ing osteopenia, reduction in hip articular space e. P hotograph of both hands at 9 years old $\mathrm{f}$. $\mathrm{P}$ hotograph of both hands at 16 years old 

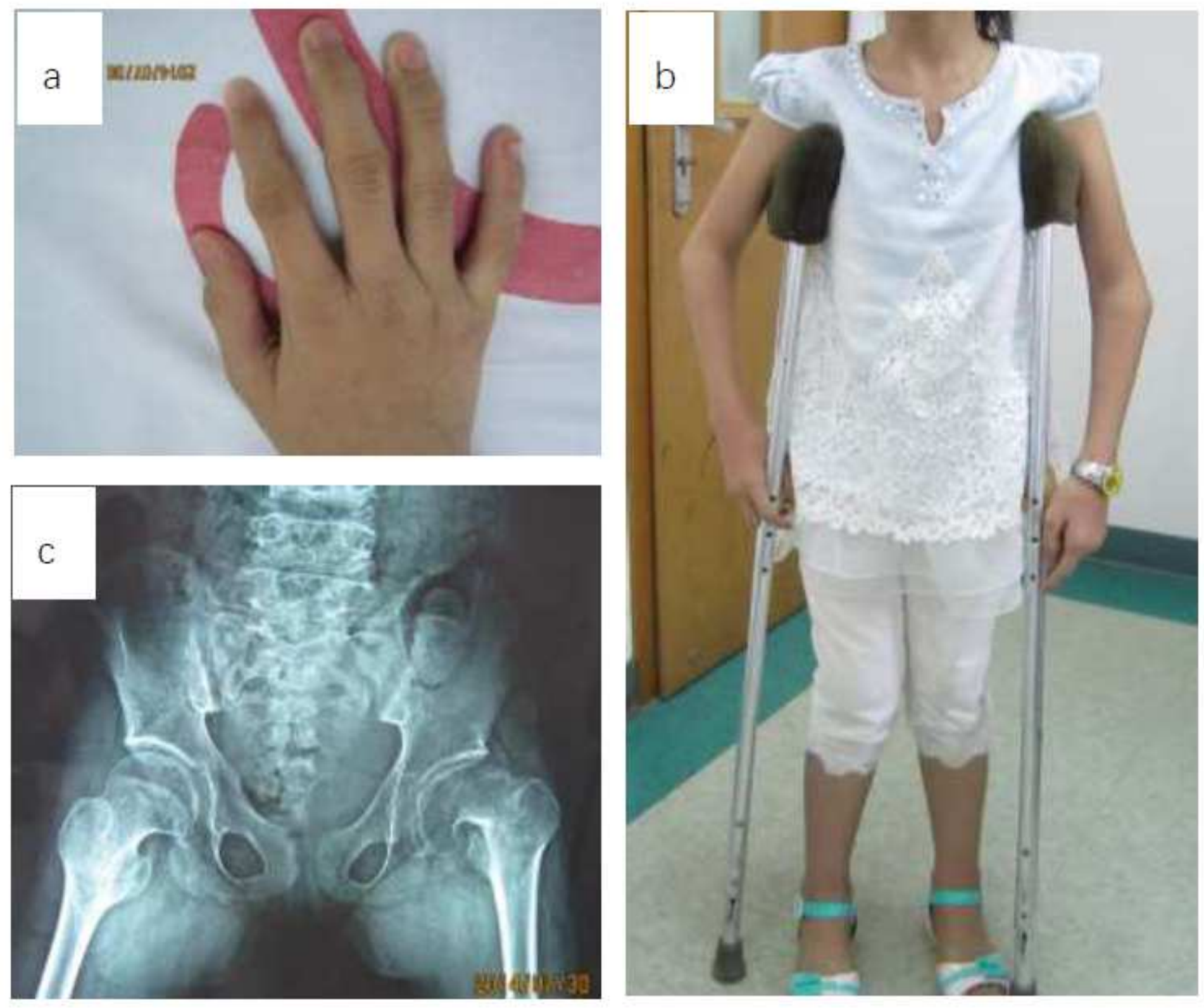

\section{Figure 3}

Images of case 3 (girl, 13 years) a. Enlarged interphalangeal joints of the fingers b. Enlarged elbows, walking difficulty and requiring crutches $\mathrm{c}$. Radiograph of pelvis showing osteopenia, reduction in hip articular space, and cyst-like structures in the left femoral head

\section{Supplementary Files}

This is a list of supplementary files associated with this preprint. Click to download.

- 2020618PPRDpapertable.pdf 